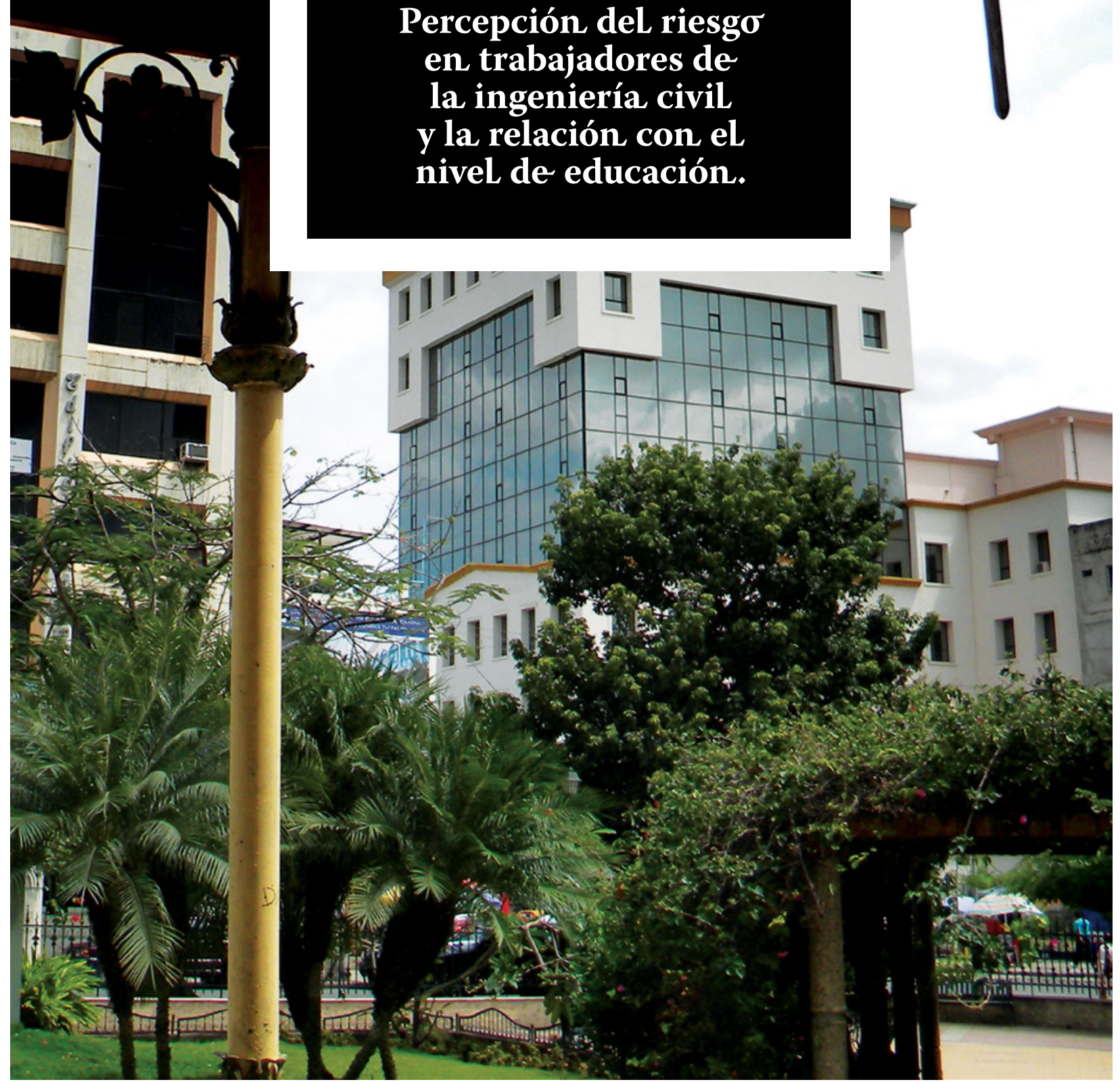




\title{
Percepción del Riesgo en TRABAJAdores de la ingeniería CiVil y LA RELACIÓN CON EL NIVEL DE EDUCACIÓN.
}

\section{PERCEPTION OF RISK IN WORIERS OF CIVIL ENGINEERING AND THE RELATIONSHIP WITH THE LEVEL OF EDUCATION.}

\begin{abstract}
RESUMEN
Los trabajadores de la ingeniería civil habitualmente están expuestos a muchos riesgos que pueden afectar su salud y la percepción de éstos no ha sido de interés para la seguridad y salud en el trabajo. El presente artículo tiene como objetivo principal analizar la percepción del riesgo laboral por parte de los trabajadores (operarios) de una empresa que realiza obras de construcciones civiles. Se planteó un estudio descriptivo transversal. El instrumento de medida es el modelo paradigma psicométrico, de esta manera se realizaron cuestionarios autoadministrados a operarios de la construcción, conservando el anonimato de los sujetos. El cuestionario constaba de nueve preguntas de dimensiones distintas acerca de la percepción de un factor de riesgo, también se añadió una pregunta sobre el nivel de educación del sujeto. El análisis de la percepción proporcionó respuestas favorables para este estudio, donde se creó la representación del perfil característico del riesgo percibido y por último se obtuvo diferencias significativas entre la percepción del riesgo con el nivel de educación básico. Este análisis refleja un enfoque importante sobre el constructo del riesgo laboral.
\end{abstract}

PALABRAS CLAVE: Nivel de educación; paradigma psicométrico; percepción del riesgo; trabajadores de la ingeniería civil.

Copyright (c) Revista San Gregorio 2019. ISSN 1390-7247; eISSN: 2528-7907 @

\begin{abstract}
The civil engineering workers are habitually exposed to many risks that can affect their health and the perception of these hasn't been of interest for occupational health and safety. The main objective of this article is to analyze this perception of the labor risk of the workers (operators) of a company that performs civil construction works. A cross-sectional descriptive study was proposed. The measuring instrument is the model of psychometric paradigm, in this way selfadministered questionnaires were made to the construction workers, preserving the anonymity of the subjects. The questionnaire consisted of nine questions of different dimensions about the perception of a risk factor, and a question about the level of education of the subject was also added. The analysis of the perception provided favorable answers for this study, where the representation of characteristic profile the characteristic profile of the perceived risk was created and finally there was significant differences between the perception of risk with basic level of education. This analysis reflects an important focus on the construct of occupational risk.
\end{abstract}

KEYWORDS: Level of education; perception of risk; psychometric paradigm; workers of civil engineering.

Copyright (c) Revista San Gregorio 2019. ISSN 1390-7247; eISSN: 2528-7907

\section{José Paúl Alcívar Rodríguez}

Geduado de Maestría de Seguridad y Salud Ocupacional "Segund

Manabí. Ecuado 


\section{INTRODUCCIÓN}

El campo laboral de la ingeniería Civil ostenta un área con un sinnúmero de peligros (Caponecchia \& Sheils, 2011) por lo que el trabajador está expuesto a factores que pueden afectar su salud, de esta forma, los accidentes se generan cuando varios de estos factores se encadenan de manera fortuita (Rodríguez, López, \& Martínez, 2013). Según Salvador (2018) los accidentes ocurren por la distracción del individuo a causa de factores emocionales.

Las características físicas y socio-demográficas del trabajador, características del trabajo, factores ambientales y factores organizacionales son puntos considerados en el proceso de investigación de accidentes. Sin embargo, los enfoques mencionados consideran los factores tangibles que afectan las prácticas seguras de trabajo.

En un estudio realizado por (Zimolong \& Trimpop, 2011) menciona que las personas a través de los sentidos perciben distintos índices de riesgo. Pero, ¿qué es un sentido y una sensación?, y ¿qué es percepción? Un sentido es (la visión, la audición, el tacto, el gusto, el olfato, el equilibrio y la orientación corporal) es una vía fisiológica particular por la que respondemos a una energía específica (estimulo). Se define como sensación a los efectos de la reacción entre los órganos sensoriales a la información captada, y percepción se concreta como la organización del cerebro para interpretar estos efectos (Papalia \& Wendkos, 1992). Así mismo, Myers (2004) define a la percepción como: "el proceso de organización e interpretación de la información sensorial, que permite reconocer el sentido de los objetos y los acontecimientos significativos" (p.231).

\section{RIESGO}

Existen varias definiciones para el riesgo. El riesgo puede ser definido como una fuente potencial de peligro o amenaza (Slovic \& Weber, 2002). Según Rodríguez et al (2015) tradicionalmente se define un peligro como: "La probabilidad del daño (cuantitativo) mientras que el riesgo se definiría como la posibilidad de que un daño ocurra (cualitativo)" (p. 258).

Faber (2002) define el riesgo como: "el producto de las posibles consecuencias de nuestras acciones y las probabilidades de que estas consecuencias ocurran" (p. 38). Así mismo, Hermansson (2012) lo define como: "algo negativo que puede suceder en el futuro" (p.18). Por otra parte, la International Organization Standardization ISO (2018) define el riesgo como: El efecto de la incertidumbre. Un "efecto" es una desviación de lo esperado (positiva o negativa) y la "incertidumbre" es el estado, incluso parcial, de deficiencia de información relacionada con la comprensión o conocimiento de un evento, su consecuente o su probabilidad (p. 6).

Así, el significado de riesgo siempre ha estado cargado de confusión y controversia (Fischhoff, Watson \& Hope, 1984), por ello Kunreuther y Slovic (1996) argumentan una visión contextualizada del riesgo.

\section{PERCEPCIÓN DEL RIESGO}

La percepción del riesgo no ha sido de mucha atención en el campo laboral de la ingeniería civil (MacDonald, 2006). Para Lion (2002) la percepción del riesgo es esencial para investigar el riesgo y según Hallowell (2010) la percepción del riesgo es el proceso de interpretación de la frecuencia y gravedad que realiza una persona ante un riesgo en particular. Así mismo, Rundmo (2000) menciona que el riesgo percibido es un juicio subjetivo que valora la probabilidad de experimentar una lesión o deterioro de la salud originado por un factor de riesgo. Sin embargo, algunos autores resaltan que el riesgo además de explicarse por su probabilidad y sus consecuencias, existen más factores que determinan su concepción.

\section{PERCEPCIÓN DEL RIESGO EN EL LUGAR DE TRABAJO}

La conducta del trabajador ante la exposición a diferentes riesgos en cierta parte depende de la percepción de los mismos. El estudio de la percepción del riesgo evalúa las acciones preventivas ejecutadas y muestran las condiciones reales de trabajo (Rundmo, 1992). Algunos autores afirman que la actitud insegura del trabajador está influenciada por 
la percepción de los riesgos (Seo, 2005); (Mohamed, Ali \& Tam, 2009). En este sentido, se ha encontrado una relación positiva entre los accidentes de trabajo y la percepción de sufrir un daño (Gucer, Oliver \& McDiarmid, 2003). Afirma Mullen (2004) que una actitud preventiva del trabajador es formada por la percepción de sufrir un daño.

La percepción de los riesgos es un factor determinante para la actitud laboral del trabajador (Stewart-Taylor \& Cherrie, 1998). El saber qué perciben los trabajadores ante los riesgos que están expuestos es de gran ayuda para encaminar la gestión de la seguridad y salud en el trabajo (Azeres \& Bizarro, 2011).

La percepción del riesgo es un enfoque poco estudiado en el campo de la ingeniería civil (MacDonald, 2006) no obstante, existen en algunas investigaciones que ha estudiado la percepción del riesgo en el campo laboral de la ingeniería civil como: Rodriguez \& López (2013) que relacionaron el riesgo percibido con el oficio que se desenvuelve el trabajador, así mismo hay un estudio exploratorio que relaciona la percepción del riesgo en los trabajadores de España y Perú (Rodríguez, Castilla \& Martínez, 2014).

En definitiva, se lleva a la necesidad de analizar la percepción del riesgo laboral y de conocer cómo los trabajadores lo perciben; ayudando a comprender la accidentabilidad en el campo laboral de la ingeniería civil y a promover una actitud preventiva de los trabajadores. Por eso, el presente trabajo intenta ser un aporte a dicho estudio de percepción del riesgo.

\section{OBJETIVO DE LA INVESTIGACIÓN}

La investigación que se realiza tiene como objetivo principal analizar la percepción del riesgo en un grupo de trabajadores (operarios) de una empresa que realiza obras de construcciones civiles en la ciudad de Portoviejo. Por lo cual se proponen los siguientes objetivos específicos: i) Crear la representación del perfil característico de la percepción del riesgo realizada por los trabajadores, (ii) Estudiar la relación del nivel de educación del trabajador en la percepción del riesgo.

\section{METODOLOGÍA}

Para llevar a cabo el objetivo de la investigación, se planteó un estudio descriptivo transversal, utilizando análisis unifactorial mediante el uso de cuestionario, el mismo que es el instrumento más frecuente en el estudio de la percepción del riesgo (Sjöberg, 1998).

Para la medición de la percepción del riesgo por los trabajadores, se eligió el modelo paradigma psicométrico. El modelo a utilizar es el resultado de una investigación desarrollada y consolidada por Paul Slovic y sus colaboradores de Decision Research de Eugene en Oregón (2000).

Esta investigación se enfoca en los 9 atributos de percepción del riesgo (A1-A9) y una pregunta de tipo global (G1) en la Nota Técnica de Prevención (NTP) 578 del Instituto Nacional de Seguridad y Salud en el Trabajo (INSST) de España realizada por Portell y Solé (2001) la misma que se basa en la investigación de Slovic (2000).

Se utilizó el procedimiento de evaluación de la percepción del riesgo desarrollada en la NTP 578; por ser un instrumento muy versátil en su aplicabilidad, es decir, su fácil adaptación en los diferentes escenarios de trabajo y por ser un instrumento ya empleado en el Ecuador (Rodríguez, Martínez \& López, 2015).

El cuestionario se estructuró atendiendo las siguientes etapas: (i) definición de los niveles de educación de los trabajadores de la variable categórica sociodemográfica "nivel de educación" (ii) selección del factor de riesgo, (iii) diseño y composición del formulario, y (iv) condiciones de aplicación.

Con la finalidad de evitar confusiones y discrepancias de los términos de la variable categórica "nivel de educación" en el cuestionario, se definieron 3 niveles de educación: básico, bachillerato y tercer nivel.

En la selección y especificación del factor de riesgo se estableció el criterio de la puntuación en la evaluación de los riesgos presentes en el área de trabajo. En esta etapa se utilizó la metodología establecida en la Guía para la identificación de los peligros y la valoración de los riesgos en seguridad y salud 
ocupacional GTC-45 (Instituto Colombiano de Normas Técnicas y Certificación, 2012).

El formulario se conformó por 9 preguntas sobre atributos del riesgo con diferentes dimensiones exploradas del paradigma psicométrico (A1-A9). Las respuestas a éstas se presentaron mediante escala de tipo Likert, con puntuaciones comprendidas entre 1 a 7 , siendo 4 el valor neutro de la escala. Adicionalmente se acompañó de una pregunta de estimación de la magnitud del riesgo (G1) después del atributo $\mathrm{A} 9$, puntuada de 0 a 100 en intervalos de 5 puntos. Las preguntas realizadas con el significado de la dimensión que explora cada atributo se exponen en la Tabla 1 , (Ver en Anexos).

La aplicación de los formularios se realizó mediante cuestionarios autoadministrados con presencia del investigador en todo momento. Con la finalidad de obtener el número de individuos necesarios para el estudio, se visitaron obras de la construcción en la ciudad de Portoviejo y se explicó a los directivos de las empresas la importancia y rentabilidad de la aplicación del formulario como herramienta preventiva ante accidentes laborales y enfermedades ocupacionales.

La población objeto de estudio se centró en trabajadores (operarios) de la construcción de una empresa. El muestreo fue por conveniencia y conservando el anonimato de los sujetos. En esta investigación se consideró que la muestra es igual a la población de estudio y se contó con $\mathrm{N}=95$ trabajadores entre peones, albañiles y maestros.

Tres cuestionarios de la muestra inicial fueron descartados por no haber sido cumplimentados en su totalidad. En tal forma la muestra final que estuvo conformada por $\mathrm{N}=92$ trabajadores de los cuales el nivel de educación fue de $32.61 \%$ básico, $64.13 \%$ bachillerato, y el $3.26 \%$ restante son de tercer nivel.

\section{ANÁLISIS Y RESULTADOS}

\section{FACTOR DE RIESGO}

Atendiendo las directrices de la GTC-45, se obtuvieron valores altos en los factores de riesgo mecánicos con puntuaciones mayores a 600 , clasificándolos como no aceptables. Para la investigación se seleccionó el factor de riesgo con mayor puntuación.
El análisis que se llevó a cabo consistió de calcular estadísticos descriptivos de la variable sociodemográfica y para cada una de las dimensiones de la percepción del riesgo; obteniendo medidas de la tendencia central y las medidas de dispersión como se muestra en la Tabla 2, (Ver en Anexos). Con estos datos se realizó el análisis de la percepción del riesgo por parte de los trabajadores.

Perfil Característico del Riesgo PERCIBIDO

Para el tratamiento de datos, se utilizó la herramienta informática IBM SPSS Statistics 25. Con las respuestas de los atributos cualitativos (A1-A9) se determinó la representación del perfil del riesgo percibido para el factor de riesgo "Mecánico". De esta manera, se construye una representación gráfica como se muestra en la Figura 1, (Ver en Anexos) obtenido de los datos de la Tabla 2.

ANÁlisis DE LA VARIANZA ENTRE EL NIVEL DE EDUCACIÓN Y LA PERCEPCIÓN DEL RIESGO.

Se realizó un análisis entre las variables "nivel de educación" y la percepción de los riesgos a través del análisis de la varianza (ANOVA), en la que pudieran determinar diferencias significativas entre los atributos de percepción del riesgo con el grupo de nivel de educación. Se realizó un análisis de la varianza entre cada uno de los 9 atributos con la variable sociodemográfica, siendo ésta la variable independiente y los atributos de percepción como variables dependientes.

Para realizar el análisis, el ANOVA parte de algunos supuestos básicos que han de cumplirse:

(1) Normalidad. Las distribuciones de probabilidad de la variable dependiente son normales. Para ello se aplicó el Teorema Central del Límite con submuestras aleatorias de tamaño 50, donde se pudo aproximar la distribución de la media a una distribución normal aplicando la prueba de normalidad de Kolmogorov-Smirnov para las variables dependientes de las submuestras aleatorias. Con el nivel de significancia o probabilidad de error de $\alpha=5 \%$ (0.05), se obtienen los p-valor para cada atributo por encima del nivel significancia, es decir, la distribución de las variables en estudio NO difiere de la distribución Normal. 
(2) Homocedasticidad. Que las varianzas de las variables dependientes en los grupos formados a partir de la variable en tratamiento (nivel de educación) sean aproximadamente iguales (homogeneidad de las varianzas). Para ello se aplicó el Test de Levene para el contraste de la homocedasticidad. Los p-valor obtenidos de la prueba y cuyos valores están por encima del nivel de significancia (0.05) por tanto, las varianzas de los grupos a comparar NO son diferentes.

(3) Independencia de los valores obtenido y aleatoriedad de la recogida muestral. La independencia de las respuestas de los sujetos fue garantizada a través de la presencia y supervisión del investigador en la recogida muestral y el suministro de los cuestionarios en todo momento, de tal forma que se evitó el incumplimiento de este supuesto.

El resultado del análisis de la varianza (ANOVA) entre la variable nivel de educación y los atributos de percepción del riesgo. Se aplica el ritual de la significancia estadística, en la que revela que la variable "nivel de educación" tiene un p-valor de 0.012 en el atributo $\mathrm{A} 1$, siendo menor al nivel de significancia $(0.05)$ existen diferencias entre los grupos.

Con la finalidad de ahondar en el resultado obtenido, se procede a realizar una prueba Post hoc. Se realiza la prueba de Tukey con el objeto de hacer comparaciones múltiples entre la variable "Nivel de educación" con los atributos de percepción. Donde no se presentaron diferencias significativas en la mayoría de los atributos (A2-A9). Sin embargo, en el atributo A1 refleja diferencias en el grupo "Básico" en relación con "Bachillerato" (p-valor $=0.022$ ).

La Tabla 3, (Ver en Anexos) muestra la clasificación de los grupos basada en el grado de parecidos existente entre sus medias en el atributo A1. Donde se refleja diferencias significativas en las medias del grupo "Básico" al no compartirse en los subconjuntos 1 y 2 . Aunque no se presente similitudes en las medias del grupo "Tercer Nivel"; no es una diferencia significativa por tener un bajo número de sujetos en relación a los otros grupos.

La Figura 2, (Ver en Anexos) se representa el perfil de las diferencias significativas de las medias entre los grupos básico, bachiller y tercer nivel en el atributo A1. Las diferen- cias solo se presentan entre los grupos básico y bachillerato, el nivel de educación tercer nivel no presenta diferencias significativas con respecto a los demás grupos.

Este resultado es acorde con las medias de la pregunta global que se realizó (G1), donde se cuantifica la magnitud del riesgo percibido por parte de los trabajadores de la ingeniería civil (Básico=72.83; Bachillerato $=80.25$; Tercer nivel= 81.67).

\section{DISCUSIÓN}

Se ha analizado la percepción del riesgo por un grupo de trabajadores (operarios) de una empresa que realiza obras de construcciones civiles en la ciudad de Portoviejo. Dicho análisis se llevó a cabo según las directrices de la Nota Técnica de Prevención NTP 578 (Portell \& Solé, 2001) y se concreta en los siguientes párrafos:

\section{PERFIL CARACTERÍSTICO DEL RIESGO PERCIBIDO OBTENIDO.}

A continuación, se comenta la implicación de los valores obtenidos de las respuestas de los trabajadores para cada atributo como se señala en la Figura 1, (Ver en Anexos).

Atributo A1. Dimensión: ConocimienTO DEL PROPIO TRABAJADOR.

El resultado de la valoración del atributo A1 (media obtenida: 5.64) es superior a 4, lo que significa que, en general el nivel de conocimiento que cree tener el trabajador sobre el riesgo mecánico es suficiente. Según Rodríguez (2013) esta premisa se puede interpretar de dos maneras: la primera pudiera ser que los trabajadores han sido formados sobre los riesgos expuestos en la construcción y la segunda sería que los trabajadores creen tener suficientes conocimientos sobre el riesgo mecánico cuando verdaderamente no lo están.

Atributo A2. Dimensión: Conocimiento del técnico de Seguridad y Salud Ocupacional (SSO).

La valoración obtenida es positiva (media obtenida: 4.37) con tendencia a la neutralidad, además, la puntuación es inferior al atributo A1. Los trabajadores perciben tener mayor conocimiento sobre los riesgos en la construcción que los técnicos en seguridad y salud ocupacional. Slovic (2000) argumenta que los 
trabajadores al estar expuestos a muchos peligros reflejan una conceptualización del riesgo más cuantiosa que la que poseen los técnicos en seguridad y salud ocupacional, aunque les falte información sobre los mismos.

\section{Atributo A3. Temor al riesgo.}

El atributo A3 (media obtenida: 5.32) profundiza la percepción al daño que puede hacerse el trabajador mientras trabaja expuesto al riesgo evaluado. La puntuación es superior a 4 lo que indica que el trabajador siente temor por hacerse daño mientras realiza su labor. Según Puy (1994) el temor es una de las variables de percepción del riesgo con mayor predicción.

Atributo A4: Vulnerabilidad del TRABAJADOR.

La puntuación obtenida en este atributo es positiva (media obtenida: 5.53 ) dando a manifiesto que el trabajador se siente vulnerable a tener un accidente mientras trabaja. Esto se debe que el trabajador haya presenciado un accidente o ha escuchado uno en su experiencia laboral.

Atributo A5: Gravedad de las ConseCUENCIAS.

El atributo A5 mide la percepción de la gravedad daño que le puede causar el factor de riesgo evaluado al trabajador. El resultado obtenido es positivo (media obtenida: 5.79) el trabajador percibe la magnitud del daño que puede afectar a su salud ante las situaciones de riesgo. Según Mullen (2004) este atributo es un índice de actitud preventiva.

\section{Atributo A6: Acción Preventiva.}

El resultado obtenido mide la percepción del trabajador para evitar que el riesgo evaluado se materialice (media obtenida: 4.39) aunque es positiva la puntuación, refleja una tendencia a la neutralidad. El trabajador cree que está en un ambiente laboral seguro cuando no es así. Thakur y Sawhney (2012) concuerdan que es necesario abordar este comportamiento cuando el trabajador decide trabajar en un entorno inseguro.

\section{Atributo A7: Acción Protectiva.}

Este atributo mide la percepción de capacidad del trabajador para reducir el impacto de daño una vez que se ha materializado el riesgo. La puntuación es positiva (media obtenida: 4.68) aunque con tendencia a la neutralidad. El trabajador cree estar indeciso ante el "puedo hacer algo" o el "no puedo hacer nada”. Leiter (2009) menciona que la formación en seguridad puede mediar la percepción de control de un riesgo.

\section{Atributo A8: Potencial catastrófico.}

El atributo A8 explora la percepción del trabajador que si el riesgo una vez que se ha materializado puede afectar a varias personas a la vez. El resultado obtenido es inferior a 4 (media obtenida: 1.77). El trabajador cree que el riesgo evaluado no puede afectar a otras personas. Esto refleja una tendencia a que el trabajador no conoce las consecuencias que podría desencadenar el riesgo. Habría que estudiar otros factores de riesgos en la construcción para realizar un análisis más exhaustivo.

Atributo A9: Demora de las ConseCUENCIAS.

El resultado obtenido en este atributo es inferior a 4 (media obtenida: 1.30 ) por lo que los trabajadores perciben que las consecuencias al riesgo mecánico pueden afectarles de manera inmediata. Varios estudios atribuyen a este elemento con menor percepción por los trabajadores (Harrell, 1990); (Mullen, 2004).

\section{RELACIÓN ENTRE EL NIVEL DE EDUCACIÓN DEL TRABAJADOR Y LOS ATRIBUTOS DE PERCEPCIÓN DEL RIESGO.}

La percepción del conocimiento propio del trabajador ante los riesgos que se encuentra expuesto es reconocido como un precursor necesario en la prevención de accidentes laborales (Seo, 2005) por lo que el nivel de educación del trabajador es un factor contribuyente en la capacidad de aprendizaje y entendimiento del mismo. De esta forma O'Connor et al. (2005) afirma que se genera un cambio en lo trabajadores a mayor formación. 
Rodríguez et al. (2015) en un estudio realizado a bomberos en la ciudad de Quito, asegura que la percepción al riesgo se incrementa a mayor formación académica. Existen otras variables que influyen en la percepción del riesgo laboral, un referente es el trabajo de Rodríguez (2013), quien encontró diferencias significativas entre el oficio y el riesgo percibido del trabajador.

\section{CONCLUSIONES}

El análisis descriptivo de la percepción del riesgo dentro de la población objeto de estudio, refleja un importante enfoque de las dimensiones del riesgo en el campo laboral de la ingeniería civil. La representación del perfil característico del riesgo percibido pone en manifiesto lo siguiente:

Atributo A1.- El conocimiento del trabajador para un riesgo, desenvuelve un rol importante en la seguridad laboral de la empresa, ya que el conocimiento erróneo o desconocimiento pudiere ocasionar accidentes con pérdidas considerables.

Atributo A2.- El trabajador al no haber sido inculcado por parte del técnico en seguridad y salud ocupacional en cuanto a los riesgos que está expuesto, crea una percepción superior del conocimiento por su experiencia laboral, en consecuencia, se puede formar un entorno laboral aparentemente seguro.

Atributo A3.- El sentir temor es esencial para la prevención del riesgo. El trabajador toma las medidas preventivas necesarias para estar seguro.

Atributo A4.- La percepción de la posibilidad de sufrir un accidente por parte del trabajador es un punto sensible para la toma de conciencia en la prevención laboral.

Atributo A5.- Cuando el trabajador tiene conocimiento acerca de la gravedad de las consecuencias causadas por un riesgo; provoca una actitud preventiva, pero cuando desconoce la magnitud del daño; el trabajador realiza actos inseguros que pudieran ocasionar un accidente.

Atributo A6.- La acción preventiva del trabajador está influenciada por los atributos anteriores, es decir, el conocimiento del riesgo, el temor al riesgo, la vulnerabilidad de su- frir un accidente y la magnitud del daño que puede causarse un trabajador ante un riesgo al que está expuesto.

Atributo A7.- Una vez que el riesgo se ha materializado, la intervención del trabajador es primordial para evitar accidentes mayores. Esto es posible con formación en seguridad y entrenamiento del trabajador ante una situación de riesgo.

Atributo A8.- La percepción del potencial catastrófico está intrínseco en la formación de seguridad ante una situación de riesgo, es decir, a mayor conocimiento del riesgo y sus consecuencias se obtiene una percepción más cuantiosa del trabajador al potencial catastrófico.

Atributo A9.- La percepción de demora de las consecuencias depende al riesgo que está expuesto el trabajador. Por ejemplo, un minero percibe que las consecuencias por realizar sus actividades pueden afectarle a largo plazo, sin embargo, el albañil lo percibe de manera inmediata por los principales riesgos al que está expuesto.

En general los resultados muestran que en obras de construcciones civiles el trabajador percibe el riesgo al que está expuesto y las consecuencias que le pudiere causar mientras realiza sus actividades.

Se ha verificado que el nivel de educación del trabajador tiene relación con la percepción del conocimiento del riesgo. Esta relación pudiera resultar una limitación en la formación en seguridad y salud a los trabajadores, ya que los trabajadores con nivel de educación básico presentan menor percepción que los que tienen bachillerato y tercer nivel.

\section{RECOMENDACIONES.}

Se necesita seguir realizando este tipo de investigaciones para ahondar otros factores que puedan afectar la percepción del riesgo, la actitud preventiva del trabajador y el ambiente laboral seguro. Un aspecto al que podría dar un valor agregado a posteriores investigaciones de percepción de riesgo, corresponde al análisis de otras variables sociodemográ- 
ficas como: sexo, edad, años de experiencia y estado civil. Con la finalidad de obtener mejores resultados y de explicar de manera más concluyente la percepción del trabajador en diferentes campos laborales.

\section{REFERENCIAS BIBLIOGRÁFICAS}

Azeres, P., \& Bizarro, M. (2011). Alcohol Consumption and Risk Perception in the Portuguese Construction Industry. The Open Occupational Health \& Safety Journal, 3, 10-17.

Caponecchia, C., \& Sheils, I. (2011). Perceptions of personal vulnerability to workplace hazards in the Australian construction industry. Journal of Safety Research, 42, 253-258.

Faber, M. (2002). Risk assessment and decision making in civil engineering. Conference Proceedings AMAS, 35-56.

Fischhoff, B., Watson, S., \& Hope, C. (1984). Defining risk. Policy Sciences, 17, 123-139.

Gucer, P., Oliver, M., \& McDiarmid, M. (2003). Workplace Threats to Health and Job Turnover Among Women Workers. Journal of Occupational and Environmental Medicine, 45(7), 683-690.

Hallowell, M. (2010). Safety risk perception in construction companies in the Pacific Northwest of the USA. Construction Management and Economics, 28(4), 403-413.

Harrell, A. (1990). Perceived Risk of Occupational Injury: Control over Pace of Work and Blue-Collar versus WhiteCollar Work. Perceptual and Motor Skills, 70(3), 13511359.

Hermansson, H. (2012). Defending the conception of "objective risk". Risk analysis : an official publication of the Society for Risk Analysis, 32(1), 16-24.

Instituto Colombiano de Normas Técnicas y Certificación. (2012). Guía Técnica Colombiana GTC45: Guía para la identificación de los peligros y la valoración de los riesgos en seguridad y salud ocupacional. Bogota: Instituto Colombiano de Normas Técnicas y Certificación (ICONTEC).

International Organization for Standardization. (2018). Sistema de gestión de la seguridad y salud en el trabajo (Vol. Primera Edición). Ginebra: ISO 2018.

Kunreuther, H., \& Slovic, P. (1996). Science, Values, and Risk. The ANNALS of the American Academy of Political and Social Science, 545(1), 116-125.

Leiter, M., Zanaletti, W., \& Argentero, P. (2009). Occupational risk perception, safety training, and injury prevention: Testing a model in the Italian printing industry. Journal of Occupational Health Psychology, 14.(1), 1-10.

Lion, R., Meertens, R., \& Bot, I. (2002). Priorities in information desire about unknown risks. Risk Analysis, $22,765-776$.

MacDonald, G. (2006). Risk perception and construction safety. Proceedings of the Institution of Civil Engineers Civil Engineering, 159(6), 51-56.

Mohamed, S., Ali, T., \& Tam, W. (2009). National culture and safe work behaviour of construction workers in Pakistan. Safety Science, 47(1), 29-35.

Mullen, J. (2004). Investigating factors that influence individual safety behavior at work. Journal of Safety Research, 35(3), 275-285.

Myers, D. (2004). Psicología. New York: Worth Publishers.
O'connor, T., Loomis, D., Runyan, C., dal Santo, A., \& Schulman, M. (2005). Adequacy of Health and Safety Training Among Young Latino Construction Workers. Journal of Occupational and Environmental Medicine, 47(3), 272-277.

Papalia, D., \& Wendkos, O. (1992). Psicología. Madrid: McGraw Hill.

Portell, M., \& Solé, M. (2001). Riesgo percibido: un procedimiento de evaluación. Instituto Nacional de Seguridad y Salud en el Trabajo. Obtenido de http://www. insht.es/InshtWeb/Contenidos/Documentacion/

FichasTecnicas/NTP/Ficheros/501a600/ntp 578.pdf

Puy, A. (1994). Percepción social del riesgo: dimensiones de evaluación y predicción. Madrid: Universidad Complutense de Madrid.

Rodríguez, I., Castilla, B., \& Martínez, M. (2014). Riesgo percibido en la construcción en España y Perú: un estudio exploratorio. Revista de la Universidad Industrial de Santander. Salud, 46(3), 277-285.

Rodríguez, I., López, M., \& Martínez, M. (2013). El riesgo percibido por el trabajador de la construcción: ; qué rol juega el oficio? Revista de la Construcción, 12(3), 83-90.

Rodríguez, I., Martínez, M., \& López, Á. (2015). El riesgo percibido y la gestión de la seguridad. Revista de la Universidad Industrial de Santander. Salud, 47, 23-32.

Rodríguez, I., Martínez, M., Delgado, A., \& Lucas, V. (2015). Factores conformantes del riesgo percibido en los trabajadores de la construcción. Dyna, 82(192), 257-265.

Rundmo, T. (1992). Risk perception and safety on offshore petroleum platforms - Part II: Perceived risk, job stress and accidents. Safety Science, 15(1), 53-68.

Rundmo, T. (2000). Safety climate, attitudes and risk perception in Norsk Hydro. Safety Science, 34, 47-59.

Salvador, J. (2018). Riesgos psicosociales del sector aeroportuario de Manta. Revista San Gregorio(22), 30-39.

Seo, D. (2005). An explicative model of unsafe work behavior. Safety Science, 43(3), 187-211.

Sjöberg, L. (1998). Risk Percepction of Alcohol Consumption. Alcoholism: Clinical and Experimental Research, 22(S7), 277s-284s.

Slovic, P. (2000). The perception of risk. London, England: Earthscan Publications.

Slovic, P., \& Weber, E. (2002). Perception of Risk Posed by Extreme Events. In: Regulation of Toxic Substances and Hazardous Waste, 2.

Stewart-Taylor, A., \& Cherrie, J. (1998). Does risk perception affect behaviour and exposure? A pilot study amongst asbestos workers. Ann Occup Hyg., 42(8), 565569.

Thakur, K., \& Sawhney, R. (2012). Analyzing perception of safety in construction workers: A cultural perspective. 62nd IIE Annual Conference and Expo 2012.

Zimolong, B., \& Trimpop, R. (2011). Risk Perception. ILO Encycl opaedia of Occupational Health \& Safety. 
ANEXOS

\begin{tabular}{ll}
\hline \multicolumn{1}{c}{ Atributo } & Dimensión explorada \\
\hline A1 & $\begin{array}{l}\text { Conocimiento del propio trabajador: Percepción del propio conocimiento que tiene el } \\
\text { trabajador sobre el riesgo evaluado }\end{array}$ \\
\hline A2 & $\begin{array}{l}\text { Conocimiento del técnico de Seguridad y Salud Ocupacional (SSO): Percepción de los } \\
\text { conocimientos que tiene el responsable de Seguridad y Salud sobre el riesgo evaluado }\end{array}$ \\
\hline A3 & Temor al riesgo: Percepción al daño que se puede hacer en el trabajo asociado al riesgo \\
& evaluado \\
\hline A4 & Vulnerabilidad del trabajador: Percepción de la vulnerabilidad \\
\hline A6 & Gravedad de las consecuencias: Percepción del daño que puede sufrir \\
\hline A7 & Acción Preventiva: Percepción de fatalidad del daño (reducir la probabilidad de aparición del \\
\hline A8 & Acción Protectiva: Percepción de control del daño (reducir el impacto del daño) \\
\hline A9 & trabajadores. \\
\hline G1 & Demora de las consecuencias: Percepción del daño a corto, mediano o largo plazo sobre del \\
& riesgo evaluado
\end{tabular}

Tabla 1. Atributos y dimensiones exploradas.

Fuente: Elaboración propia a partir de Slovic (2000) y Portell y Solé (2001).

\begin{tabular}{|c|c|c|c|c|c|c|}
\hline & $\mathrm{N}$ & Mínimo & Máximo & Media & $\begin{array}{l}\text { Desviación } \\
\text { estándar }\end{array}$ & Varianza \\
\hline $\begin{array}{l}\text { A1: Conocimiento del } \\
\text { propio trabajador }\end{array}$ & 92 & 4.00 & 7.00 & 5.641 & 0.909 & 0.826 \\
\hline $\begin{array}{l}\text { A2: Conocimiento del } \\
\text { técnico de SSO }\end{array}$ & 92 & 3.00 & 6.00 & 4.370 & 0.808 & 0.653 \\
\hline A3: Temor al riesgo & 92 & 4.00 & 7.00 & 5.315 & 0.662 & 0.438 \\
\hline $\begin{array}{l}\text { A4: Vulnerabilidad del } \\
\text { trabajador }\end{array}$ & 92 & 4.00 & 7.00 & 5.533 & 0.999 & 0.999 \\
\hline $\begin{array}{l}\text { A5: Gravedad de las } \\
\text { consecuencias }\end{array}$ & 92 & 5.00 & 7.00 & 5.793 & 0.655 & 0.429 \\
\hline A6: Acción Preventiva & 92 & 3.00 & 7.00 & 4.391 & 1.109 & 1.230 \\
\hline A7: Acción Protectiva & 92 & 3.00 & 6.00 & 4.685 & 0.824 & 0.680 \\
\hline $\begin{array}{l}\text { A8: Potencial } \\
\text { catastrófico }\end{array}$ & 92 & 1.00 & 5.00 & 1.772 & 0.743 & 0.552 \\
\hline $\begin{array}{l}\text { A9: Demora de las } \\
\text { consecuencias }\end{array}$ & 92 & 1.00 & 2.00 & 1.304 & 0.463 & 0.214 \\
\hline
\end{tabular}

Tabla 2. Estadísticos descriptivos.

Fuente: Elaboración propia. 
HSD Tukey ${ }^{\mathrm{a}, \mathrm{b}}$

\begin{tabular}{|c|c|c|c|}
\hline \multirow[b]{2}{*}{ Nivel de educación } & \multirow[b]{2}{*}{$\mathrm{N}$} & \multicolumn{2}{|c|}{ Subconjunto para alfa $=0.05$} \\
\hline & & 1 & 2 \\
\hline Básico & 30 & 5.2667 & \\
\hline Bachillerato & 59 & 5.7966 & 5.7966 \\
\hline Tercer Nivel & 3 & & 6.3333 \\
\hline Sig. & & 0.457 & 0.448 \\
\hline
\end{tabular}

Se visualizan las medias para los grupos en los subconjuntos homogéneos.

a. Utiliza el tamaño de la muestra de la media armónica $=7,820$.

b. Los tamaños de grupo no son iguales. Se utiliza la media armónica de los tamaños de grupo.

Tabla 3 HSD Tukey - Atributo A1

Fuente: Elaboración propia.

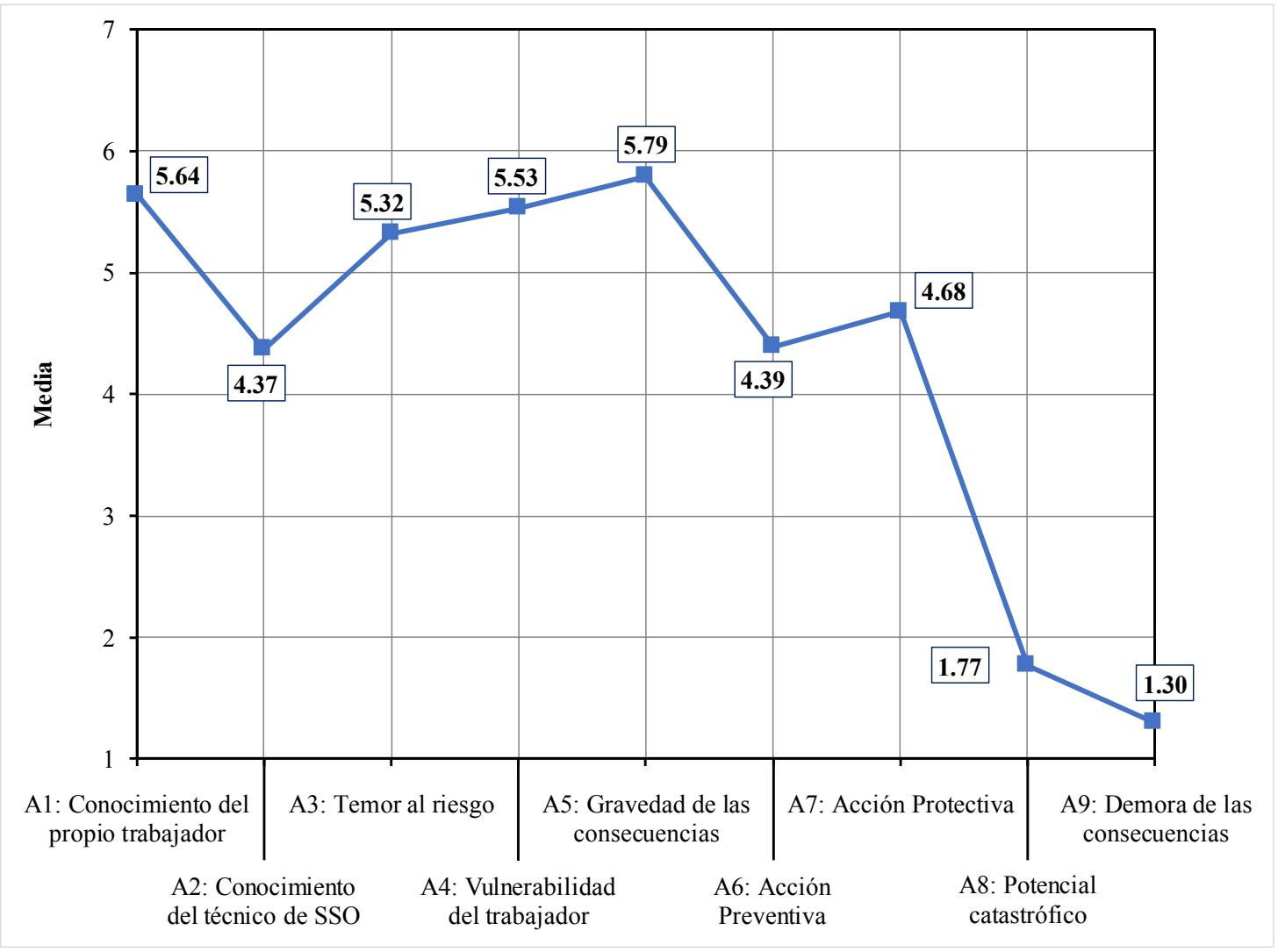

Figura 1. Perfil característico del riesgo percibido "Mecánico".

Fuente: Elaboración propia. 


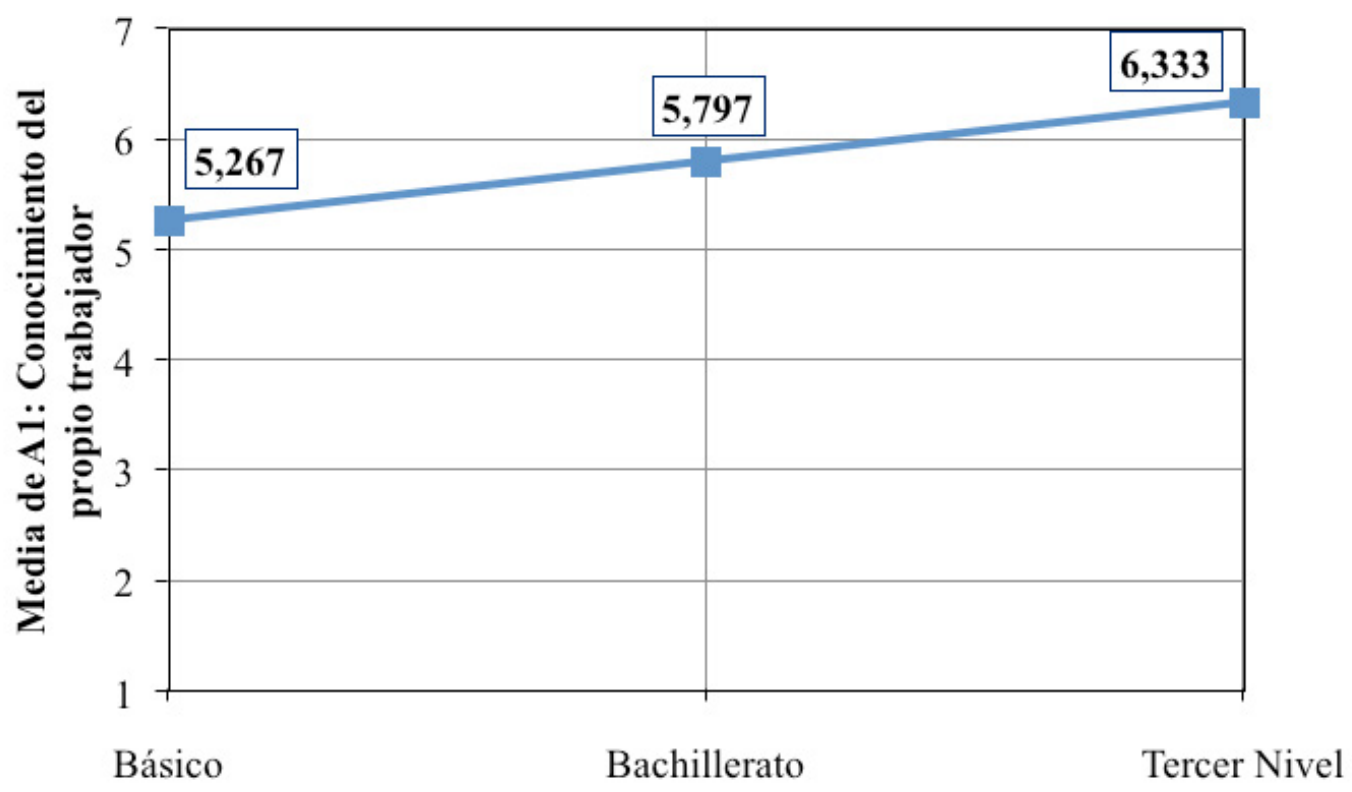

Figura 2. Relación entre la variable "Nivel de educación" y el atributo A1 Fuente: Elaboración propia. 